\title{
The Influence of an Acute Bout of High-Intensity Interval Exercise on Heart Rate Variability Indices and Stress Index in the Absence of Cardiometabolic Diseases \\ Original Research
}

Riccardo Torres ${ }^{1}$, Kathleen A. Richardson ${ }^{1}$, Catherine Lowry ${ }^{2}$, Cassidy Beeson ${ }^{1}$, Ahmed Ismaeel ${ }^{3}$, Panagiotis Koutakis ${ }^{3}$, and Jeffrey S. Forsse ${ }^{1}$.

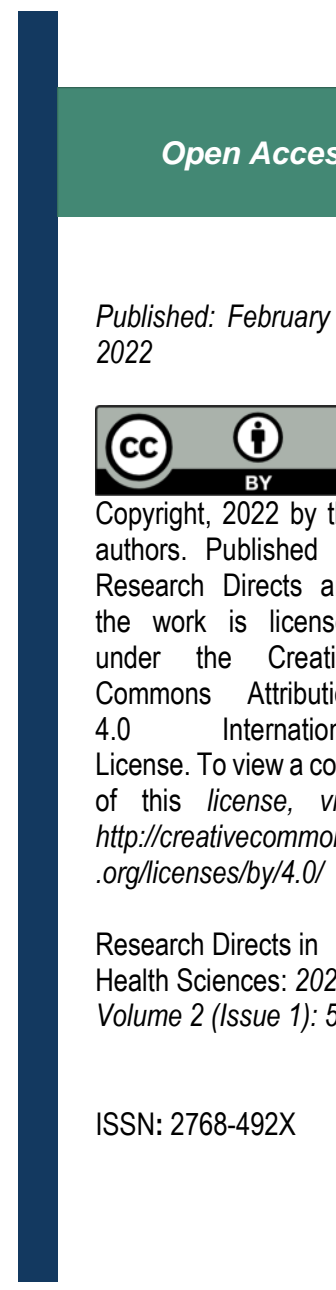

\author{
${ }^{1}$ Department of Health, Human Performance, and Recreation, Baylor University; Waco, TX; \\ ${ }^{2}$ Department of Health and Exercise Science, Colorado State University, Fort Collins, CO \\ ${ }^{3}$ Clinical Muscle Biology Lab, Baylor University, Waco, TX.
}

\begin{abstract}
Introduction: The maximal rate of oxygen consumption is the gold standard when determining cardiorespiratory fitness (CF) in healthy and diseased populations. $\mathrm{CF}$ has been shown to influence the improvement of cardiac autonomic modulation (CAM) and lower the risk of morbidity and mortality rates. High-intensity interval exercise (HIIE) has been shown to influence CAM post-exercise in various populations. Heart rate variability (HRV) is a non-invasive way to assess CAM during, before, and after exercise. The purpose was to determine if a single acute bout of HIIE is influenced by age and $\mathrm{CF}$ when correlated to HRV variables to determine CAM in healthy and fit individuals.

Methods: Thirty-four healthy individuals ( $\mathrm{n}=21$ male; $\mathrm{n}=13$ female) completed a single acute bout of HIIE session to quantify CAM via HRV. HRV was assessed pre, 1-hour, and 24-hours post-exercise using time and frequency domains, and stress index (SI).

Results: HRV time and frequency domains were not significantly changed. The SI was significantly different between pre, 1-hour, and 24hours post-exercise $(p=0.001)$. The SI was also significantly different between age groups $(p=0.025)$.

Conclusion: The lack of significant difference in CAM can be attributed to the participants high CF, which helps maintain their HRV as they age. Additionally, the SI appears to be a good metric to assess CF as individuals age.
\end{abstract}

Key Words: heart rate variability, stress index, high-intensity interval exercise

Corresponding author: Jeffrey S. Forsse, Jeff_Forsse@Baylor.edu

\section{Introduction}

Heart rate variability (HRV) is the difference in time between each corresponding heartbeat ${ }^{1}$. HRV is a non-invasive way to assess the parasympathetic nervous system by charting the variation in time between each heartbeat, representing the dysregulation of the autonomic nervous system (ANS) and sympathetic nervous system (SNS) ${ }^{2}$. Currently, HRV can be used as a tool to predict health outcomes, such as sudden cardiac death, cardiovascular disease 
(CVD), and numerous chronic illnesses that may subsequently arise form ANS and SNS dysfunction ${ }^{1}$. HRV is not only used to evaluate the health conditions of at-risk populations, but it can also be used to assess the health and performance of athletes ${ }^{3}$. HRV focuses on time and frequency domains, and utilizes high frequency (HF), low frequency (LF), NN intervals and R-R intervals that can be used to determine ANS/SNS relationship and the influence on morbidity and mortality ${ }^{4}$. Another aspect that is also monitored in conjunction with HRV is stress index (SI), which demonstrates how well the heart and ANS react to physiological stress ${ }^{5}$.

HRV is known to be influenced by physical activity $(\mathrm{PA})$ and exercise in healthy and diseased populations ${ }^{1}$. In general, individuals who regularly participate in aerobic exercise have a lower HRV response to PA in comparison to less physically active individuals ${ }^{6}$. When comparing moderate-to-high-intensity aerobic exercise, high-intensity has a greater effect on cardiac neuromuscular activity ${ }^{7}$. Over the last few decades, high-intensity interval exercise (HIIE) has become more commonly used in elite athletes, healthy individuals, and diseased populations ${ }^{8-10}$. HIIE consists of repeated bouts of high-intensity exercise followed by short recovery periods of low-intensity exercise with higherintensity exercise causing a lower HRV response ${ }^{6}$. HRV has also been used to help assess recovery time post-exercise 11. Individuals who perform more HIIE have demonstrated a better recovery time compared to individuals who do not preform HIIE ${ }^{12}$. While research on this topic is limited, studies have demonstrated that HIIE can provide positive results on HRV in at-risk populations ${ }^{13}$. For example, HIIE has been shown to improve cardiovascular health which can promote higher HRV and overall longevity ${ }^{13}$.

As individuals age, the amount of exercise and/or PA decreases due to a variety of factors such as lethargy, lack of time, and proper resources, leading to the deterioration of overall health ${ }^{14}$. Physiological changes begin to manifest in HRV measurements which have been shown to decline with age, with the most drastic changes occurring between 20 to 30 years of age ${ }^{1}$. Variations in HRV measurements have demonstrated that even healthy individuals can still display ANS dysfunction as a result of aging ${ }^{2}$. Various studies have demonstrated improvements in resting HRV following aerobic exercise ${ }^{4}$. The purpose of this study was two-fold: 1) to examine the effect an acute bout of HIIE on HRV, and 2) to document the effect of age has on HRV. Our primary hypothesis is that significant changes in HRV will occur in the hours following a single acute bout of HIIE. Our secondary hypothesis is there will be no significant difference in HRV between younger vs older individuals following HIIE.

\section{Scientific Methods}

Prior approval for the study was obtained by the local university Institutional Review Board (IRB) for research with human subjects (project \#1574622-3). The study protocol conforms to the ethical guidelines of the 1975 Declaration of Helsinki as reflected in approval granted by the institution's human research committee. Eligible individuals were provided both verbal and written information regarding the research study prior to consent. Participants signed and returned the informed consent document and underwent further health screening before admittance into the study.

\section{Subjects}

Participants were between 20 to 60 years of age who were physically active (achieving the minimum exercise recommendations established by the American Heart Association and the American College of Sports Medicine), nonsmokers, non-obese, having never been diagnosed with cardiovascular or metabolic diseases, and were currently not taking any medication except vitamins. Participants were required to regularly visit their primary care physician to rule out medical diagnoses. Participants were excluded if they were diagnosed with any form of CVD, diabetes, dyslipidemia, cancer, or any other chronic health disparity. A total of 57 individuals expressed interest in the study; 23 were excluded due to having one or more cardiometabolic diseases. Thirty-four ( $\mathrm{n}=21 \mathrm{men} ; \mathrm{n}=13$ women) healthy individuals were admitted into the research study. Participant demographics are provided in Table 1.

\section{Study Screening Procedures}

Participants arrived at the lab after a minimum four-hour fast from calorie-containing substances; water consumption during this time was encouraged. Participants were told to abstain from exercise for 24-hours before coming to the lab to undergo body composition, DEXA test, and $\mathrm{VO}_{2 \max }$ testing. Participants were asked to wear shorts, a t-shirt, and a pair of comfortable jogging/running shoes. All of the experimental procedures were reviewed again with each participant, and any questions they had concerning the study were answered. 
Table 1. Participant Demographics.

\begin{tabular}{lc}
\hline Variables & \\
\hline Age (yrs.) & $37.6 \pm 12.2$ \\
Height (inches) & $68.0 \pm 3.7$ \\
Weight (lbs.) & $161.3 \pm 33.1$ \\
Body Fat (\%) & $19.2 \pm 7.0$ \\
$\mathrm{VO}_{2 \mathrm{max}}\left(\mathrm{ml} / \mathrm{kg} / \mathrm{min}^{-1}\right)$ & $41.8 \pm 7.3$ \\
Heart Rate $(\mathrm{bpm})$ & $60 \pm 11.3$ \\
Resting SBP $(\mathrm{mmHg})$ & $116.9 \pm 7.0$ \\
Resting DBP (mmHg) & $77.2 \pm 6.0$ \\
\hline All values are presented as mean \pm standard deviation. \\
Abbreviations: DBP = Diastolic blood pressure; \\
SBP = Systolic blood pressure; and $\mathrm{VO}_{2}=$ oxygen uptake.
\end{tabular}

Baseline Assessment

Baseline height and weight were determined using an electronic scale and stadiometer (Seca 703). The participants were asked to lay on a table for five minutes to record resting HRV and heart rate. Blood pressure was obtained using a manual standing sphygmomanometer. All measurements throughout the test were performed by trained exercise physiologists.

\section{Body Composition Assessment}

Waist circumference was measured at the level of the umbilicus with a cloth tape and was measured three times to ensure accuracy. Participants underwent a standard dual x-ray absorptiometry (DXA) scan, to determine body fat percentage and bone density.

HRV Data Collection

HRV was recorded using an elastic electrode Polar belt (H7TM, Polar Wearlink ${ }^{\circledR}$ Lake Success, NY), which was placed on the distal third of the sternum. HRV data was collected on all three visits and taken on three time points (baseline, 1-hr, and 24-hrs post-exercise). In the preliminary screening visit the participants remained seated for 5 minutes to collect resting HRV data. HRV was then recorded during the entire $\mathrm{VO}_{2 \text { max }}$ test. During the HIIE intervention, HRV was collected at three times points; immediately prior to exercise, 1-hr post-exercise, and 24-hrs post-exercise. For each assessment, participants were instructed to rest quietly in the supine position while HRV data was collected for five minutes. Time and frequency domain HRV variables were measured and reported in Table 2 . The stress index was also measured at all three time points using the CardioMood equation $\left(\mathrm{SI}=[\right.$ Amplitude of Mode $\%] /\left[2 * \mathrm{Mode}^{*}\left(\mathrm{RR}_{\max }\right.\right.$ $\left.-\mathrm{RR}_{\min }\right){ }^{10}$.

Table 2. HRV time and frequency domain definitions.

\begin{tabular}{|l|l|}
\hline High Frequency (HF) & $\begin{array}{l}\text { Measures } 0.15-0.40 \mathrm{~Hz} \text { and reflects parasympathetic } \\
\text { activity which corresponds to HR variations related to } \\
\text { the respiratory cycle }\end{array}$ \\
\hline Low Frequency (LF) & $\begin{array}{l}\text { Measured 0.04-0.015 Hz and reflects baroreceptor } \\
\text { activity during resting conditions where both sympathetic } \\
\text { and parasympathetic activity can be observed }\end{array}$ \\
\hline L/H Frequency Ratio (LF/HF) & $\begin{array}{l}\text { Measured at 0.0033-0.04 Hz and is associated with all- } \\
\text { cause mortality (e.g., low VLF is an indicator for } \\
\text { arhythmic death) }\end{array}$ \\
\hline HF/VLF Frequency Ratio (HF/VLF) & $\begin{array}{l}\text { Measures the ration of LF to HF which is intended to } \\
\text { observe the ratio between SNS to PNS activity }\end{array}$ \\
\hline
\end{tabular}




\begin{tabular}{|l|l|}
\hline Total Power (TP) & The combined power of HF, LF, and VLF \\
\hline RMSSD & $\begin{array}{l}\text { The root mean square difference between normal } \\
\text { heartbeats; reflects the beat-to-beat variance }\end{array}$ \\
\hline SDNN & $\begin{array}{l}\text { The standard deviation of normal sinus beats (NN } \\
\text { Intervals) and is the gold standard for medical } \\
\text { stratification of cardiac risk }\end{array}$ \\
\hline pNN50 & $\begin{array}{l}\text { The percentage of adjacent NN intervals that differ from } \\
\text { each other more than 50ms and is correlated with } \\
\text { peripheral nervous system }\end{array}$ \\
\hline WN4 & $\begin{array}{l}\text { The width of the histogram taken at the level of } 4 \% \text { of } \\
\text { the total count of R-R intervals }\end{array}$ \\
\hline
\end{tabular}

Exercise Testing

Each participant performed a maximal aerobic exercise treadmill test to determine $\mathrm{VO}_{2 \max }$ and maximal heart rate, allowing for cardiorespiratory fitness classification. Respiratory gases $\left(\mathrm{VO}_{2}\right.$ and $\left.\mathrm{VCO}_{2}\right)$ were measured continuously using a mouthpiece and headgear connected to an integrated respiratory gas analysis system (TrueOne $2400^{\mathbf{T M}}$, ParvoMedics ${ }^{\circledR}$, Sandy, UT). Respiratory gases and heart rate were continuously monitored throughout the test. The exercise test began with one minute of standing to obtain resting respiratory values. The treadmill speed was determined using the participant's $5 \mathrm{~K}$ race pace. Speed was increased every three min for the first three stages. The incline grade was at $0 \%$ for the first three stages and was increased by $2 \%$ increments every subsequent minute, with the speed remaining constant after stage three until the end-of-test criteria were reached ${ }^{17}$. To achieve a true $\mathrm{VO}_{2 \max }$, individuals were required to obtain a respiratory exchange ratio (RER) of $>1.1$, a plateau of $\mathrm{VO}_{2}$, and/or achieve $85 \%$ or higher of age-predicted max heart rate ${ }^{18}$. The test was terminated if the researchers observed signs or symptoms that warrant termination, or at the participant's request.

\section{Exercise Session}

A minimum of a two-day washout period, with an average of 13.1 days between screening and HIIE session, was implemented following the $\mathrm{VO}_{2 \max }$ test ${ }^{15,16}$. Participants could then return to the test site to perform the HIIE experimental condition. The experimental exercise condition consisted of a single 30-minute bout of HIIE that was completed in five-minute intervals of exercise. There were six sets of intervals consisting of three minutes of submaximal, yet vigorous, exercise at $80 \%$ of the individual's peak $\mathrm{VO}_{2} \mathrm{Reserve}\left(\mathrm{VO}_{2} \mathrm{R}\right)$, followed by two minutes of walking at $40 \%$ of their $\mathrm{VO}_{2} \mathrm{R}$ (Figure 1). During the entire exercise session, heart rate and $\mathrm{VO}_{2} \mathrm{R}$ levels were continuously monitored.

\section{High Intensity-Interval Exercise (HIIE)}

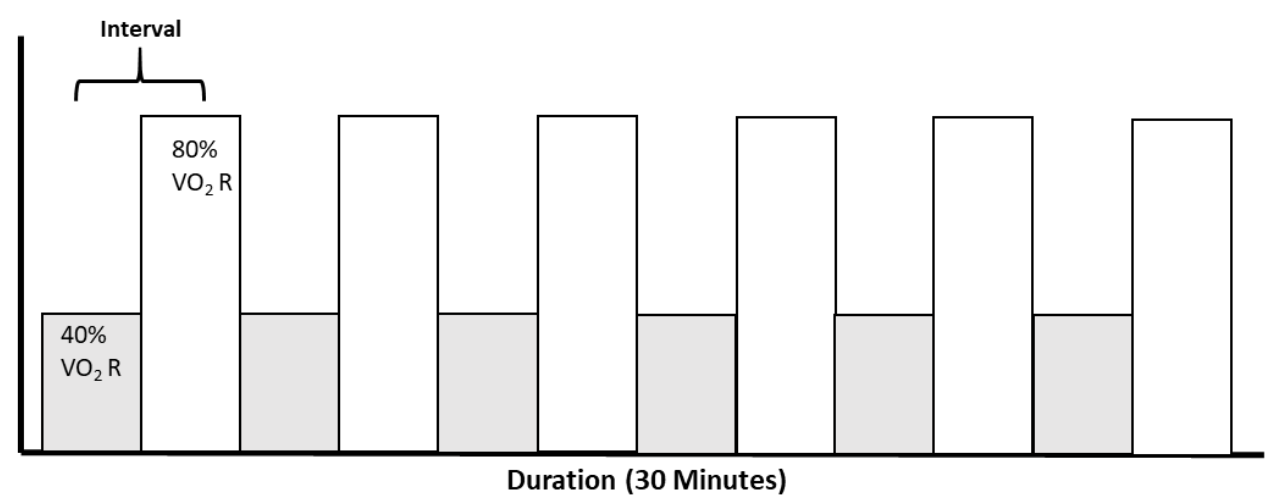

Figure 1. Exercise protocol.

There were six sets of intervals consisting of three minutes of submaximal exercise at $80 \%$ of the individual's peak $\mathrm{VO}_{2} \mathrm{R}$, followed by two minutes of walking at $40 \%$ of

Results their $\mathrm{VO}_{2} \mathrm{R}$.

Cardiorespiratory fitness was not significantly different between age groups $(p=0.096) . \mathrm{VO}_{2 \max }$, on average, was 43.7 \pm 7.4 in the younger group, and $39.5 \pm 6.9$ in the older group. Time domain pNN50 was significantly different 
between the younger and older groups $(p=0.002)$ but was not significantly different between time points regardless of age group (Figure 2). There were no other significant changes in time or frequency domains at 1-hour or 24-hour time points for age, time, or age by time. There were no differences in gender, or differences in gender across time.

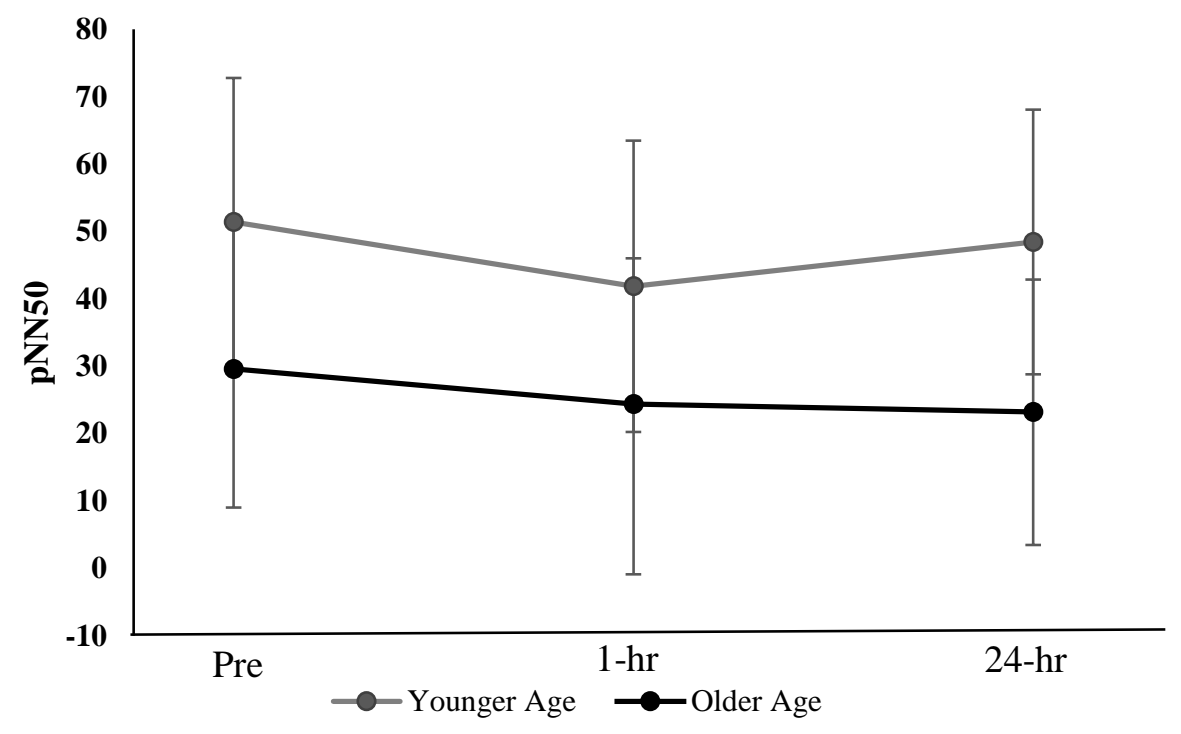

Figure 2. pNN50 Over Time.

All data is presented as mean \pm SE.

The SI overall was significantly different between pre, 1-hour, and 24-hour post-exercise $(p=0.001)$ (Figure 3). The SI was also significantly different between age groups $(p=0.025)$. However, there was no interaction effect of time and age $(p=0.615)$. There was a negative correlation observed between cardiorespiratory fitness and SI $(r=-0.117 ; p$ $=0.537$ ) indicating that higher $\mathrm{VO}_{2}$ resulted in a lower SI pre- and post-exercise (Figure 4).

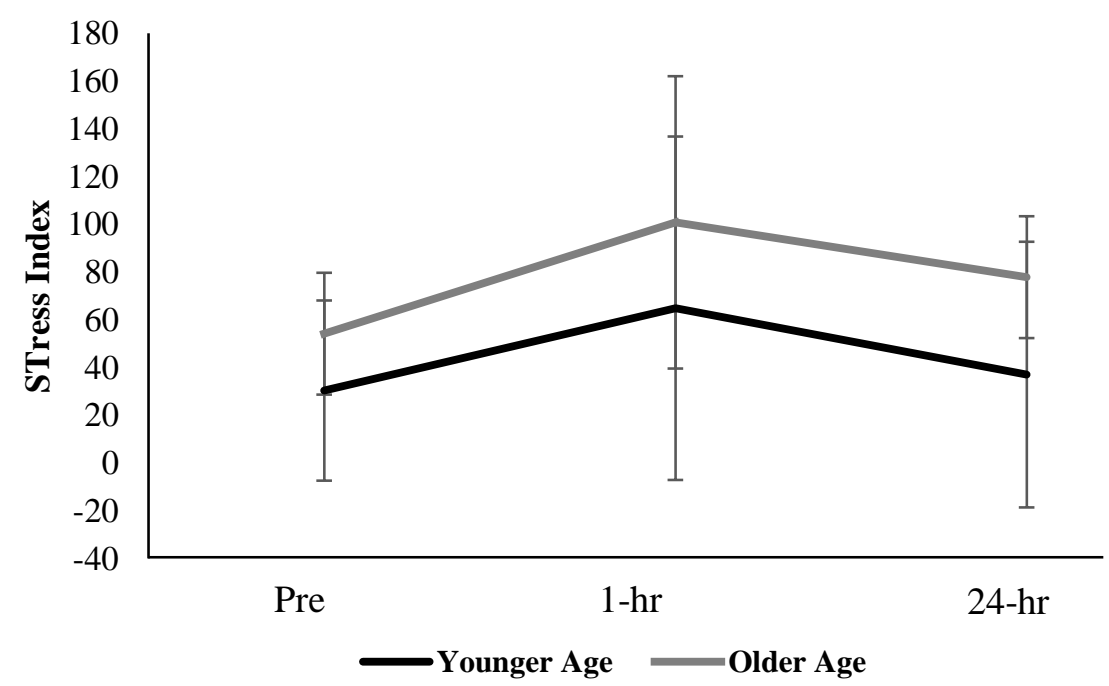

Figure 3. Stress Index Over Time. All data is presented as mean $\pm \mathrm{SE}$. 


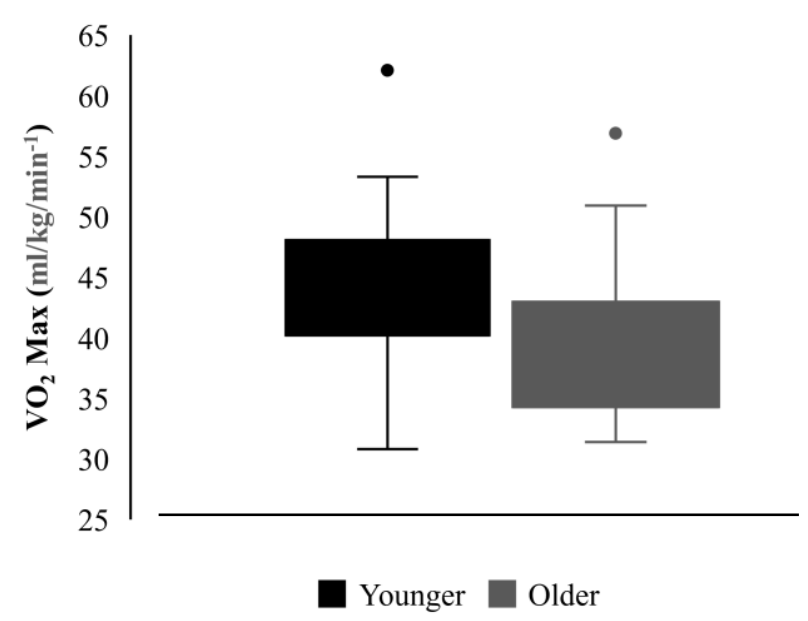

Figure 4. $\mathrm{VO}_{2 \max }$ in Younger vs. Older Groups. All data is presented as mean $\pm \mathrm{SE}$. - = Significant difference between younger and older individuals, $p<0.05$.

\section{Discussion}

The focus of this study was to get a better understanding of the effects that a single acute bout of HIIE had on HRV across different age groups with the absence of cardiometabolic diseases by analyzing time and frequency domains in conjunction with the SI. Significant differences in the SI were observed across timepoints $(p=0.001)$, between age groups $(p=0.025)$, and there was a higher negative correlation between SI and cardiorespiratory fitness levels when using $\mathrm{VO}_{2}$ max. These differences demonstrate that healthy individuals can maintain a higher level of HRV as they age and manage the stress of exercise more effectively. Time domain pNN50 demonstrated significance when assessing the effect of age $(p=0.002)$. The remaining time and frequency domains of HRV demonstrated no significance differences across all three time points in either young or older age groups.

Physiological stress has a distinctive effect on the nervous system, which affects both the peripheral nervous system (PNS) and the ANS. Both systems are critical in communicating properly with the immune system, and dysregulation of both nervous systems can cause improper communication with the immune system and other major issues ${ }^{17}$. Using the stress index can give insight into the inner workings of the PNS and ANS systems during, before, and post-exercise and can be used as an indicator for abnormal cardiac responses ${ }^{5}$. The differences observed between the SI in the two age groups in Figure 3 demonstrate a change in the ANS and PNS activity as individuals age. Despite the older group having higher overall SI values, the SI increased and decreased simultaneously, and a similar rate of change as the younger group. This finding indicated that older individuals had a similar rate of decline in the SI from baseline to 24 hours as the younger individuals. The effect of a higher SI level is concurrent with other studies that suggest that ANS/PNS activities are affected negatively as we age ${ }^{2,18}$. Our results may signify that even though older individuals have damped PNS/ANS functions, they still function as they should when placed under physiological stress, which helps with overall longevity and cardiac health. The positive effect exercise has on the heart can also be seen in Figure 4, which demonstrates an inverse relationship between $\mathrm{VO}_{2 \max }$ values and $\mathrm{SI}$ levels. The individuals with a higher $\mathrm{VO}_{2 \max }$ tend to get closer or reach baseline values 24-hours after the acute bout of HIIE when compared to those with lower $\mathrm{VO}_{2 \max }$ levels, which remained slightly elevated at 24-hours post-exercise. These results support maintaining higher CF levels to have a better ANS/PNS function that can not only help performance but also help with recovery and prevent ANS/PNS function from deteriorating. As seen in previous studies, individuals with better fitness tend to have higher HRV values. Thus, higher CF levels provide individuals with the necessary ANS adaptations to quickly change their heart rate in response to the exercise stimulus ${ }^{11}$. After subjecting the body to the stress of exercise, recovery time is vital for athletes and coaches, and it can be achieved using the SI to avoid overtraining. While not many studies have reported SI values, it would be a beneficial variable to explore further and would provide a noninvasive assessment in conjunction with HRV to the general population, athletes, and coaches. 
From the time domain variables that were measured in this study, pNN50 was the only variable that was significantly different across time. The pNN50 value quantifies the percentage of adjacent NN intervals that differ by more than $50 \mathrm{~ms}$, and is correlated with PNS activity ${ }^{1}$. This variable is also correlated with RMSSD and HF, that are also used to asses PNS function ${ }^{1,19}$. While there was no significance in the RMSSD, this does not mean that there was a discrepancy, instead this could be due to the inconsistency of maximal HR and weak correlation with these time domain variables ${ }^{1}$. Along with that inconsistency, the lack of variation in the other time domain variable can also be attributed to the similarity in fitness levels of the participants. In previous studies done with athletes and fit individuals, there were no variations in time domain variables, even though their protocols used moderate-intensity exercise for the workout regiments. Though these exercise protocols were different from our current exercise protocol, there is valuable information regarding the relationship between fitness and time domain variables ${ }^{3}$. Claiborne et al. ${ }^{20}$ did a large scale study of 176 collegiate athletes and obtained HRV values at rest. The HRV data provided insightful information regarding HRV variables in relation to having low CVD risk factors and comparing pNN50, HF, LF, and $\mathrm{LF} / \mathrm{HF}$ between aerobic and anerobic athletes. ${ }^{20}$. The key takeaway from our study is the changes noted in pNN50 throughout the experiment. As seen in Figure 4 the older individuals had a lower pNN50 when compared to the younger individuals, throughout the duration of the experiment. These results can be an indicator of how age continues to affect ANS function regardless of fitness or overall health. As individuals age, the PNS undergoes changes multiple times with decreases beginning to occur in the 20's and then around the age of 60 it appears to increase again ${ }^{2}$. Even in healthy individuals there is a decline in PNS activity, but the decline appears to be less when vigorous weekly exercise is involved ${ }^{21}$.

The frequency domains displayed no changes among all the variables that were measured, but this does not reflect a lack of information. Instead, the results show that among healthy populations there is less variance in the time domain variable due to healthy PNS/SNS function. In previous studies that looked at the effects of exercise on frequency domain generally there were no changes observed, which are primarily thought to be due to the complexity of the parasympathetic and sympathetic interactions, which are still not fully established ${ }^{22}$. When specifically looking at the relationship between PNS/SNS, the ratio of $\mathrm{HF} / \mathrm{LF}$ is most highly correlated with the nervous system ${ }^{1}$. Exercise was observed to elicit a change in untrained individuals, specifically in $\mathrm{HF} / \mathrm{LF}$, which demonstrated a small decrease in the sympathetic activity and an increase in the parasympathetic activity after high intensity interval training (HIIT) ${ }^{14}$. The change demonstrates that exercise continues to improve the cardiac ANS by allowing more parasympathetic activity following the bout of exercise to bring the body back down to baseline levels. Although, a change was not noted in our study, further research is needed to better understand specific timepoint frequency domain variables in response to exercise since there have been many contradictory results as to what level of exercise intensity causes an increase in $\mathrm{LF}$ or an increase in $\mathrm{HF}^{22}$. Despite the contradictory information regarding frequency domain variables, there is sound evidence from healthy populations that exercise promotes better ANS function ${ }^{20}$.

Our study, while novel, has limitations. First, the lab setting, a controlled environment in which the environmental conditions are different from real-life environment, could have caused changes in HRV that may have been different if observed outside the lab. Second, the acute bout of HIIE that was used in this study did not provide us with long term results of how HIIE could affect HRV based on the 24-hr time limit.

Practical applications from this study include 1) utilizing the SI value more frequently since it is easy to understand for the public and provides valuable information for practitioners. 2) observing and recording the amount of variation certain training regimens have on fit individuals since healthy and active individuals tend to have low amounts of variation. 3) Recovery time needs to be adjusted for individuals depending on their fitness level, by account of $\mathrm{VO}_{2 \max }$ levels. This is based on the relationship that was observed between $\mathrm{VO}_{2 \max }$ and $\mathrm{SI}$ levels, where individuals with higher $\mathrm{VO}_{2 \max }$ levels recovered better after 24 hours.

\section{Conclusions}

In conclusion, a single acute bout of HIIE was not successful in altering HRV time or frequency domains in healthy individuals. However, age remained a factor in CAM, regardless of fitness, based on pNN50 levels and SI levels that varied between younger and older participants. The SI also demonstrated that more time may be needed to recover after a bout of HIIE, especially for those individuals with a lower $\mathrm{VO}_{2 \max }$ level. While $\mathrm{HRV}$ has been studied for many years, the practical implications of it as an assessment marker are still very much unexplored, and therefore further research is needed to observe the prolonged effects of exercise on HRV, such as HIIE or continuous moderateintensity exercise. Along with reporting SI values, more focused investigation into the reliability and practicality of SI is needed. 


\section{Acknowledgements}

The authors would like to thank the participants for their time and effort.

\section{References}

1. Shaffer F, Ginsberg JP. An Overview of Heart Rate Variability Metrics and Norms. Front Public Health. 2017;5:258. doi:10.3389/fpubh.2017.00258

2. Geovanini GR, Vasques ER, de Oliveira Alvim R, et al. Age and Sex Differences in Heart Rate Variability and Vagal Specific Patterns - Baependi Heart Study. Glob Heart. 2020;15(1):71. doi:10.5334/gh.873

3. Sherman SR, Holmes CJ, Hornikel B, MacDonald HV, Fedewa MV, Esco MR. Heart-Rate Variability Recording Time and Performance in Collegiate Female Rowers. Int J Sports Physiol Perform. 2021;16(4):550-556.

4. Sessa F, Anna V, Messina G, et al. Heart rate variability as predictive factor for sudden cardiac death. Aging. 2018;10(2):166-177. doi:10.18632/aging.101386

5. Kim HG, Cheon EJ, Bai DS, Lee YH, Koo BH. Stress and Heart Rate Variability: A Meta-Analysis and Review of the Literature. Psychiatry Investig. 2018;15(3):235-245. doi:10.30773/pi.2017.08.17

6. Michael S, Graham KS, Davis GMO. Cardiac Autonomic Responses during Exercise and Post-exercise Recovery Using Heart Rate Variability and Systolic Time Intervals-A Review. Front Physiol. $2017 ; 8$. doi:10.3389/fphys.2017.00301

7. Ramírez-Vélez R, Tordecilla-Sanders A, Téllez-T LA, et al. Effect of Moderate- Versus High-Intensity Interval Exercise Training on Heart Rate Variability Parameters in Inactive Latin-American Adults: A Randomized Clinical Trial. J Strength Cond Res. 2020;34(12):3403-3415. doi:10.1519/JSC.0000000000001833

8. Chang YM, Shiao CC, Huang YT, et al. Impact of metabolic syndrome and its components on heart rate variability during hemodialysis: a cross-sectional study. Cardiovasc Diabetol. 2016;15(1):16. doi:10.1186/s12933016-0328-2

9. Peterson MN, Forsse JS, Papadakis Z, Grandjean PW. Heart Rate Variability Responses To Exercise In Midspectrum Chronic Kidney Disease: 940 Board \#66 May 27 2:30 PM - 4:00 PM. Med Sci Sports Exerc. 2020;52(7S):230. doi:10.1249/01.mss.0000676064.81595.b1

10. Papadakis Z, Forsse JS, Peterson MN. Effects of High-Intensity Interval Exercise and Acute Partial Sleep Deprivation on Cardiac Autonomic Modulation. Res Q Exerc Sport. Published online August 25, 2020:1-19. doi:10.1080/02701367.2020.1788206

11. Makivić B, Nikić Djordjević M, Willis MS. Heart Rate Variability (HRV) as a Tool for Diagnostic and Monitoring Performance in Sport and Physical Activities. J Exerc Physiol Online. 2013;16(3):103-131.

12. Panissa VLG, Cal Abad CC, Julio UF, Andreato LV, Franchini E. High-Intensity Intermittent Exercise and its Effects on Heart Rate Variability and Subsequent Strength Performance. Front Physiol. 2016;7:81. doi:10.3389/fphys.2016.00081

13. Alansare A, Alford K, Lee S, Church T, Jung HC. The Effects of High-Intensity Interval Training vs. ModerateIntensity Continuous Training on Heart Rate Variability in Physically Inactive Adults. Int J Environ Res Public Health. 2018;15(7):E1508. doi:10.3390/ijerph15071508

14. Ramírez-Vélez R, Tordecilla-Sanders A, Téllez-T LA, et al. Effect of Moderate- Versus High-Intensity Interval Exercise Training on Heart Rate Variability Parameters in Inactive Latin-American Adults: A Randomized Clinical Trial. J Strength Cond Res. 2020;34(12):3403-3415. doi:10.1519/JSC.0000000000001833

15. Javorka M, Zila I, Balhárek T, Javorka K. Heart rate recovery after exercise: relations to heart rate variability and complexity. BrazJ Med Biol Res. 2002;35:991-1000. doi:10.1590/S0100-879X2002000800018

16. Gifford RM, Boos CJ, Reynolds RM, Woods DR. Recovery time and heart rate variability following extreme endurance exercise in healthy women. Physiol Rep. 2018;6(21):e13905. doi:10.14814/phy2.13905

17. Marques AH, Silverman MN, Sternberg EM. Evaluation of Stress Systems by Applying Non-invasive Methodologies: Measurements of Neuroimmune Biomarkers in the Sweat, Heart Rate Variability and Salivary Cortisol. Neuroimmunomodulation. 2010;17(3):205-208. doi:10.1159/000258725

18. Garavaglia L, Gulich D, Defeo MM, Thomas Mailland J, Irurzun IM. The effect of age on the heart rate variability of healthy subjects. PLOS ONE. 2021;16(10):e0255894. doi:10.1371/journal.pone.0255894

19. Mietus JE, Peng CK, Henry I, Goldsmith RL, Goldberger AL. The pNNx files: re-examining a widely used heart rate variability measure. Heart. 2002;88(4):378-380.

20. Claiborne A, Alessio H, Slattery E, Hughes M, Barth E, Cox R. Heart Rate Variability Reflects Similar Cardiac Autonomic Function in Explosive and Aerobically Trained Athletes. Int J Environ Res Public Health. 2021;18(20):10669. doi:10.3390/ijerph182010669 
21. Felber Dietrich D, Schindler C, Schwartz J, et al. Heart rate variability in an ageing population and its association with lifestyle and cardiovascular risk factors: results of the SAPALDIA study. Eur Eur Pacing Arrbythm Card Electrophysiol J Work Groups Card Pacing Arrbythm Card Cell Electropbysiol Eur Soc Cardiol. 2006;8(7):521-529. doi:10.1093/europace/eul063

22. Michael S, Graham KS, Davis GM. Cardiac Autonomic Responses during Exercise and Post-exercise Recovery Using Heart Rate Variability and Systolic Time Intervals-A Review. Front Physiol. 2017;8:301. doi:10.3389/fphys.2017.00301 